many social determinants of health. NEs can be conceptualised as differing from other research in three important ways. First, unlike trials, investigators cannot allocate the exposure of interest which is instead externally decided. Second, researchers are unable to influence the exposure's characteristics which results in uncertainty of the fidelity, dose and whether the NE will occur at all. Third, and in contrast to many observational studies, the timing of the exposure and hence research itself, cannot be chosen by researchers.

Methods Using three case studies of research on H1N1 (two quantitative sero-epidemiology studies, one qualitative study) we identified key factors that allowed us to successfully conduct our investigation of a NE. We compared these factors with the wider NE literature to identify common barriers and facilitators to research. Results We identified key external factors that influence capacity to successfully take advantage of NEs. As a result of the uncertainty of a NE occurring and the rapid response required, flexibility is needed by researchers, policymakers, practitioners, ethics committees and funders. This is achievable when research is perceived as immediately important for health (as in H1N1) but may otherwise be difficult.

Discussion We suggest researchers investigating NEs face additional challenges to traditional observational studies. While some barriers are insurmountable, actions such as rapid response funding, fasttrack ethics procedures and improvements in routine data can create a more conducive environment allowing policy-relevant evaluation

\section{P1-187 ACCESS TO ALCOHOL OUTLETS AND HARMFUL ALCOHOL CONSUMPTION: A MULTILEVEL STUDY IN MELBOURNE, AUSTRALIA}

doi:10.1136/jech.2011.142976d.80

${ }^{1} \mathrm{~A}$ Kavanagh, ${ }^{2} \mathrm{M}$ Kelly, ${ }^{1} \mathrm{~L}$ Krnjacki, ${ }^{3} \mathrm{~L}$ Thornton, ${ }^{4} \mathrm{D}$ Jolley, ${ }^{5} \mathrm{~S} \vee$ Subramanian, ${ }^{6} \mathrm{G}$ Turrell, ${ }^{1} \mathrm{R}$ Bentley. ${ }^{1}$ The University of Melbourne, Victoria, Australia; ${ }^{2}$ La Trobe University, Victoria, Australia; ${ }^{3}$ Deakin University, Victoria, Australia; ${ }^{4}$ Monash University, Victoria, Australia; ${ }^{5}$ Harvard University, Boston, USA; ${ }^{6}$ Oueensland University of Technology, Queensland, Australia

Introduction While developed countries have either introduced, or are considering, legislation to restrict the number of alcohol outlets, there is little evidence to support this strategy except in the USA.

Methods Multilevel study of 2334 adults aged 18 to 75 years from 49 census collector districts (the smallest spatial unit in Australia at the time of survey) in Melbourne, Australia. Alcohol outlet density was defined as the number of outlets within a one kilometre road network of respondents' homes and proximity was the shortest road network distance to the closest outlet from their home. Using multilevel logistic regression we estimated the association between outlet density and proximity and four measures of harmful alcohol consumption: drinking at levels associated short-term harm at least weekly and monthly; drinking at levels associated with long-term harm and frequency of consumption.

Results Density of outlets was associated with increased risk of alcohol related harm with the strongest association evident for drinking at levels associated with short term harm at least weekly (OR 1.10, 95\% CI 1.04 to 1.16). When density was fitted as a categorical variable, the highest risk of drinking at levels associated with short-term harm was when there were eight or more outlets (short-term harm weekly: OR 2.36, 95\% CI 1.22 to 4.54 and shortterm harm monthly: OR $1.80,95 \%$ CI 1.07 to 3.04$)$. We found no evidence to support an association between proximity and harmful alcohol consumption.

Conclusion Restricting the number of off-premise alcohol outlets is likely to reduce levels of harmful alcohol consumption.

\section{P1-188 ANALYSIS OF QUESTIONNAIRE SURVEYS ON MOTHERS' ANXIETIES ABOUT CHILD-REARING IN RURAL AREAS OF JAPAN}

doi:10.1136/jech.2011.142976d.81

${ }^{1} \mathrm{H}$ Kawasaki, ${ }^{2} \mathrm{M}$ Nlshiyama, ${ }^{3} \mathrm{M}$ Fujita, ${ }^{3} \mathrm{P}$ D'Angelo, ${ }^{1} \mathrm{~S}$ Moriwaki. ${ }^{1}$ Hiroshima University, Hiroshima, Japan; 'Hiroshima Bunkyo Women's University, Hiroshima, Japan; ${ }^{3}$ Doi Clinic, Hiroshima, Japan

Background In Japan, as society changes, becoming ever more isolated and with rises in nuclear families, mothers are increasingly concerned and uneasy about child care. These changes have also coincided with a significant decrease in birth rates. The government took out the "indicator of support plan for the 21st century" in 2001. So, each prefecture had to draw up a concrete plan by 2005 .

Purpose The purpose of study was to explore factors affecting mothers' anxieties and to evaluate effects of a child care support plan. Sample and Statistical Analysis The questionnaire surveys were conducted with 1000 mothers, selected randomly, in 2002 and 2008. Mothers' anxiety was measured using feelings of loneliness, confusion about child-rearing and lack of standards as the children's development process. These were analysed using "ordered categorical logistic regression". The explanatory variables were mother's age, child's age and child-rearing methods.

Results 'Feelings of loneliness' was negatively correlated with a child's age ( $p$ value $=0.07$ ) and a maternal age $(p$ value $=0.044)$. Mothers' mental instability and a lack of families' cooperation significantly related with 'feelings of loneliness' ( $p$ values $=0.000$, 0.000 , respectively). "Feelings of loneliness" and "confusion about child-rearing" in 2008 (after implementation of the child care support plan) were reduced compared with those in 2002 ( $p$ values $=0.069,0.065$, respectively).

Conclusions The introduction of a child care support plan including opportunities for counselling, friendship and activities increased family co-operation and more social interaction for both mother and child. The research shows these can be effective methods in reducing mothers' anxieties.

\section{P1-189 NET BENEFIT FRACTION AS A MODIFIED POPULATION ATTRIBUTABLE FRACTION TO EVALUATE THE CLINICAL PERFORMANCE OF A RISK FUNCTION}

doi:10.1136/jech.2011.142976d.82

${ }^{1,2} \mathrm{D}$ Khalili, ${ }^{*}{ }^{1} Y$ Mehrabi, ${ }^{2} \mathrm{M}$ Bozorgmanesh. ${ }^{1}$ Department of Epidemiology, School of public health, Shahid Beheshti University of Medical Sciences, Tehran, Iran; ${ }^{2}$ Prevention of Metabolic Disorders Research Center, Research Institute for Endocrine Sciences, Shahid Beheshti University of Medical Sciences, Tehran, Iran

Introduction Net benefit (NB) has been suggested to verify the clinical usefulness of a model beyond its discrimination and calibration but it has a complex meaning in prediction models. Here we define net benefit fraction (NBF) using Population Attributable Fraction (PAF) index.

Methods According to Vickers and Elkin (2006), in models that predict diseases to treat high risk individuals, NB is defined as true positive (TP) rate minus weighted false positive (wFP) rate; the weight is the odds at the threshold probability for treatment $(\mathrm{Pt} /[1$ $\mathrm{Pt}]$ ). Dividing NB by its maximum, incidence of disease, gives the portion of incidence prevented by the treatment. A shortcoming of $\mathrm{NB}$ is that it assumes the treatment reduces the incidence in TPs to zero. On the other hand, PAF is defined as reduction of the risk to below the threshold, that is, to that of low risk population. However, PAF does not take into account the false positives. Therefore, we suggest NBF calculated as PAF minus wFP rate, the latter divided by incidence. We applied the method to calculate the clinical performance of Framingham risk function at routine 
threshold probability of $20 \%$ to predict cardiovascular diseases in a population based cohort of 6224 Iranians aged 30-74 years with 10year follow-up.

Results dividing NB by incidence resulted $17 \%$ and PAF shows $43 \%$ decrease in incidence, but NBF shows just $8 \%$ advantage for treatment according to the model.

Conclusion NBF seems to be a challengeable issue in policy making using risk functions.

\section{P1-190 SOCIAL DETERMINANTS OF CARDIAC DISEASE BIOMARKERS: INVESTIGATING A SWEDISH MALE COHORT AT AGES 50 AND 70}

doi:10.1136/jech.2011.142976d.83

A Khanolkar, * D Vågerö, I Koupil. Centre for Health Equity Studies, Karolinska Institutet/ Stockholm University, Stockholm, Sweden

Background Social status is associated with cardiovascular disease (CVD) prevalence and incidence. We aimed to study relationships between i) socioeconomic position (SEP) and common CVD biomarkers; cholesterol, LDL/HDL, ApoB/ApoA1 and adiponectin ii) SEP and CVD mortality in a Swedish-population-based sample, and to assess if these associations changed with age.

Design A longitudinal cohort study of men born 1920-24 with clinical measurements, blood samples, questionnaire data and register-based information on SEP and cause of death.

Methods Of 2322 men that participated in an investigation at age 50, 1221 attended a reinvestigation at age 70. SEP was measured as occupational class and educational level. Linear regression (adjusted for age, body mass index and physical activity) was used to study associations between SEP and CVD biomarkers. CVD mortality over 36 year's follow-up was analysed by Cox regression.

Results At age 50: We found significant inverse associations of education and occupational group with mean cholesterol levels, whereas LDL/HDL ratio was associated with education only. These were statistically significant after adjustment for covariates. No significant associations were found between either measure of SEP and $\mathrm{ApoB} / \mathrm{ApoA} 1$ ratio. At age 70: No significant associations were found between either measurement of SEP and any biomarker studied. Men classified as highest educated and non-manual had decreased risk for CVD mortality during follow-up.

Conclusions Associations of SEP with cholesterol levels and LDL/ $\mathrm{HDL}$ ratio that exist at age 50, are no longer found in the same group of men at age 70 . We found no significant association between SEP and adiponectin levels at age 70 .

\section{P1-191 DO USA STATE-LEVEL SOCIAL SPENDING AND INCOME INEOUALITY PREDICT INDIVIDUAL MORTALITY? A FIXED EFFECTS, INSTRUMENTAL VARIABLE ANALYSIS}

doi:10.1136/jech.2011.142976d.84

${ }^{1,2}$ D Kim. ${ }^{*}{ }^{1}$ RAND Corporation, Santa Monica, California, USA; ${ }^{2}$ Harvard School of Public Health, Boston, Massachusetts, USA

Introduction Government spending on public goods (eg, education) and social assistance (eg, cash transfers) provides plausible investments in the social determinants of health. Among rich nations, countries with higher social spending and lower income inequality show longer life expectancies. However, studies of both factors have been limited by bias from residual confounding and reverse causation. Methods This study examined data from the National Longitudinal Mortality Study on 431637 adults aged 30-74 in 48 USA states followed for 11 years. State per capita social spending (total, welfare, education, health) and income inequality (Gini coefficient) were explored as predictors of individual mortality (all-cause, cardiovascular, cancer) using linear probability models. To reduce bias, models incorporated state and time fixed effects and instrumental variables, and controlled for state- and individual-level covariates.

Results Total public spending and spending on welfare and education, but not healthcare, predicted lower probabilities of death from coronary heart disease (CHD) (per $\$ 250$ per capita spent on welfare: $\beta=-0.016, p=0.03$ ) and all causes combined (per $\$ 250$ per capita spent on welfare: $\beta=-0.031, p=0.03$ ). There were weaker associations for colon cancer and no associations for stroke. A higher Gini coefficient predicted a higher probability of CHD mortality but not all-cause mortality. Stratified models suggested stronger effects among those aged 45-59 and with incomes $<\$ 25000 /$ year.

Conclusion Higher state social spending outside the healthcare sector may reduce one's chances of dying from heart disease and all causes combined, particularly for low-income, middle-aged adults. Policies promoting economic equality may further lessen $\mathrm{CHD}$ disparities.

\section{P1-192 A SMALL AREA ANALYSIS OF INJURIES AND RELATED FACTORS: 2009 COMMUNITY HEALTH SURVEY, KOREA}

doi:10.1136/jech.2011.142976d.85

${ }^{1} \mathrm{Y} \mathrm{M}$ Kim, ${ }^{*}{ }^{2} \mathrm{H}$ Hong, ${ }^{2} \mathrm{~J} \mathrm{H} \mathrm{Lim},{ }^{3} \mathrm{~K}$ Cheong, ${ }^{3} \mathrm{~T} \mathrm{H}$ Yoon. ${ }^{1}$ Department of Preventive Medicine, Dong-A University College of Medicine, Busan, Republic of Korea; ${ }^{2}$ School of Public Health, Seoul National University, Seoul, Republic of Korea; ${ }^{3}$ Department of Preventive Medicine, Pusan National University, Busan, Republic of Korea

Introduction As the incidence and death rate of injury are relatively high in Korea, the public health concerns are now increasing. The aim of this study are (1) to describe the regional distribution and pattern of the injury prevalence, (2) to evaluate the relationship between the Korea poverty index and the regional variation of the injury.

Methods <2009 Korea Community Health Survey> was the national interview survey in Korea. The type of injury was categorised by ICECI and the age adjusted injury prevalence was calculated. The regional variation was evaluated by the index; EO (Extremal Quotient), CV (Coefficient of Variation), CVA (CV from ANOVA). For explaining the effect of Korea poverty index to regional variation, general linear model was applied.

Results The 1-year experience of all kinds of injury was more prevalent to men than to women. Falls $(30 \%)$ was most frequent type of injury. The significant regional variation was found according to the category of injury. In the urban area, the 1-year experience rate of all kinds of injury was 5.47 (4.39-6.54) in poorest region by Korea poverty index, and 4.61 (4.28-4.94) in richest region. The statistically significant linear trend was found across the quartile of poverty index ( $p$ for trend $=0.04$ ).

Discussion There were significant regional variations according the rurality, administrative regions, and the Korea poverty index. For the tackling the high prevalence and regional discrepancy of the injury, community specialised and area based intervention were needed.

\section{P1-193 TWO-YEARS MORTALITY AFTER EVIDENCE BASED DRUG THERAPY OF ACUTE MYOCARDIAL INFARCTION: A POPULATION-BASED COHORT STUDY IN ROME, ITALY}

doi:10.1136/jech.2011.142976d.86

U Kirchmayer, ${ }^{*}$ V Belleudi, N Agabiti, M D Martino, L Bauleo, L Pinnarelli, D Fusco, M Arcà, M Davoli. Department of Epidemiology of the Regional Health Service, Lazio Region, Italy

Introduction Clinical guidelines recommend treatment with evidence-based (EB) drug therapy (antiplatelets, $\beta$-blockers, agents acting on the renin-angiotensin system, statins) for patients with acute myocardial infarction (AMI). Results on long term outcomes from observational population studies are scarce.

Objectives Analysing the association between EB drug therapy and 2 years mortality in AMI patients. 